\title{
Immune-Related Prognostic Model in Colon Cancer: A Gene Expression-Based Study
}

\author{
Haojie Yangt, Wei Jin', Hua Liu, Dan Gan, Can Cui, Changpeng Han* and Zhenyi Wang* \\ Department of Coloproctology, Yueyang Hospital of Integrated Traditional Chinese and Western Medicine, Shanghai \\ University of Traditional Chinese Medicine, Shanghai, China
}

Mounting evidence supports that the malignant phenotypes of cancers are defined not only by the intrinsic activity of tumor cells but also by immune cells that are recruited and activated in tumor-related microenvironment. Here, we developed a diagnostic and prognostic model for colon cancer, based on expression profiles of immune-related genes and immune cell component. As a result, we found that B cell infiltration ratio, CD4+ T cells, as well as immune-related genes of TRIB3, CHGA, CASP7, LGALS4, LEP, NOX4, IL17A, and HSPD1 may be highly relevant with clinical outcome of colon cancer.

Edited by:

Jie Sun,

Wenzhou Medical University, China

Reviewed by: Nitish Kumar Mishra,

University of Nebraska Medical

Center, United States

Shaoli Das,

National Institutes of Health (NIH),

United States

*Correspondence:

Changpeng Han

13918314533@163.com

Zhenyi Wang

yyyygangchangke@163.com

${ }^{\dagger}$ These authors have contributed equally to this work

Specialty section:

This article was submitted to Bioinformatics and Computational

Biology,

a section of the journal

Frontiers in Genetics

Received: 20 November 2019

Accepted: 30 March 2020

Published: 08 May 2020

Citation:

Yang H, Jin W, Liu H, Gan D,

Cui C, Han C and Wang Z (2020)

Immune-Related Prognostic Model

in Colon Cancer: A Gene

Expression-Based Study.

Front. Genet. 11:401.

doi: 10.3389/fgene.2020.00401
Keywords: colon cancer, bioinformatics, immune-related gene, immune cell infiltration, prognostic model

\section{INTRODUCTION}

Colon cancer is the third most common type of cancer, making up $\sim 10 \%$ of all cases (Forman et al., 2014), especially in developed countries, where more than $65 \%$ of cases have been found. It was reported that $\sim 145,290$ new cases of colorectal cancer were diagnosed every year in the United States (Kim et al., 2018). In spite of the advances in screening, diagnosis, and curative resection, colon cancer is still one of the leading causes of cancer death worldwide, with unsatisfactory clinical outcome (Siegel et al., 2017). Presently, the molecular pathogenesis of this cancer is still poorly understood.

Recently, mounting evidence supports that the malignant phenotypes of cancers are defined not only by the intrinsic activity of tumor cells but also by immune cells that are recruited and activated in tumor-related microenvironment (Ben-Baruch, 2002). A previous study reported that tumor can be viewed as distinct immunological organ, which has complex immune microenvironment (Fridman et al., 2012). Pathologists have long recognized the diversity of immune infiltration into tumors, and the most widely studied are tumor-infiltrating lymphocytes (TILs) (Bense et al., 2017). TILs have been suggested to promote or inhibit tumor growth actively and are important to the clinical outcome (Brockhoff et al., 2018; Shibutani et al., 2018; Udall et al., 2018). In terms of colon cancer, study has demonstrated that the level of lymphocyte infiltration into primary tumor is a strong independent predictor of overall survival (OS). What is more, high lymphocyte infiltration represents a positive prognostic factor (Galon et al., 2006). In addition to immune cells, the cancer tissues also include various chemokines, cytokines, and growth factors (Bremnes et al., 2011). They interact with each other to form tumor-related microenvironment and exert inhibitory effects on tumor cells. Tosolini et al. (2011) have applied expression profiling of colorectal cancer to define the relevance of specific immune signatures and found that T helper 17 (Th17) type, interleukin (IL)17-dominant immune profiles indicated a poor prognosis, and Th1 type, interferon- $\gamma$-dominant immune profiles indicated an improved prognosis.

In this study, we developed a molecular classifier associated with colon cancer prognosis based on the gene expression profiles of immune-related genes. This study may provide insights 
into the complex relationship between the heterogeneity of immune cells and disease prognosis in colon cancer.

\section{RESULTS}

\section{Differential Expression Analysis}

On the basis of adj. $p$-value $<0.05$ and $|\operatorname{logFC}|>1$, we identified 265 differentially expressed (200 upregulated and 65 downregulated) immune genes. The volcano plot of all differentially expressed immune genes and the heatmap of top 10 up- and downregulated immune genes are shown in Figure 1.

\section{EPIC Immune Cell Infiltration Ratio}

Figure $2 \mathrm{~A}$ shows infiltration ratio of immune cells. Other cells, including endothelial cells, $\mathrm{CD}^{+} \mathrm{T}$ cells, and CD8+ $\mathrm{T}$ cells accounted for a relatively large proportion, while $\mathrm{B}$ cells, carcinoma-associated fibroblasts (CAFs), macrophages, and natural killer (NK) cells accounted for a relatively small proportion. For the infiltration ratio of each immune cell, Welch two-sample non-paired $t$-test was used to conduct statistical analysis of tumor vs. normal samples, and the results showed that there were significant differences $(p<0.001)$ except for NK cells (Figure 2B).

\section{Diagnostic Model of Colon Cancer Based on Immune Characteristics}

With immune cells component and the expression profile of differentially expressed immune genes being features for model input, the integrated sample number was 329 (288 tumor and 41 normal), and the feature number was 272 (265 differentially expressed immune genes and the composition ratio of seven immune cells except for NK cells). Dimensionality reduction in principal component analysis (PCA) was performed on the feature dimensions of each sample, and the results showed that the tumor and normal samples were significantly distinguished when only the first two principal components were used (Figure 3A), indicating that these features could be well used to construct a diagnostic model.

After cross-validation of the training model, we obtained the optimal parameters of different models and the evaluation results on the test set, as shown in Table 1. These models were excellent predictors of colon cancer samples, both in terms of accuracy and area under the curve (AUC) (the AUC values of SVM, L1-LR, and RandomForest were 1) (Figure 3B). Through the XGBoost model, we ranked the importance of features and selected the top 20 features (Figure 3C), including TRIB3, CHGA, multiple LGALS family genes (LGALS4, LGALS3, LGALS2, and LGALS9), CASP7, B cell infiltration ratio, etc.

\section{Immune Gene Expression, Immune Cell Component, and Survival Analysis}

The downloaded clinical TCGA data contained phenotypic information of 551 samples, and 277 samples had both gene expression profile and effective survival information. We extracted survival status and survival time of these samples and plotted K-M survival curve by combining the expression of 265 differentially expressed immune genes in different samples, immune cell component, and sample clinical information. The significance threshold was set as $p<0.05$. The results showed that $\mathrm{CD} 4^{+} \mathrm{T}$ cells and 72 immune genes were highly relevant to prognosis. Survival curves of $\mathrm{CD} 4^{+} \mathrm{T}$ cells and the top five immune genes are shown in Figure 4 based on the significance of $p$-value.

\section{Clinical Phenotype and Survival Analysis}

In line with the survival status and survival time of 551 samples in TCGA clinical data, we drew K-M survival curves in combination with gender, age, histological type, number of lymph nodes, tumor, node, and metastasis (TNM) stage, colonic polyp, and prognosis information (a total of 244 valid samples). The significance threshold was set as $p<0.01$. The results showed that the number of lymph nodes (lymph node examined count, $p=0.0067$ ), $M$ staging (pathologic $M, p=0.00011$ ), and $T$ staging (pathologic T, $p<0.0001$ ) were connected with survival time (Figure 5).

\section{Prognostic Model of Colon Cancer Based on Immune Characteristics}

Using "train_test_split" package in Python, we divided 277 samples with both gene expression profile and effective survival information into training set and test set at a ratio of 5:5 (random state $=123$ ). Considering the content of $\mathrm{CD}^{+} \mathrm{T}$ cells and the expression values of 72 immune genes, we used Cox multivariate regression to construct a prognostic model. The results of both likelihood ratio test and score (log-rank) test were significant, and the $p$-values were $p=1.493 e-08$ and $p=0.001621$, indicating that this multivariate model was highly relevant to the prognosis. In Cox multivariate model, there were 19 factors $(p<0.05)$ of great influence, including CD $4^{+} \mathrm{T}$ cells, LEP, NOX4, RETNLB, LAIR1, IL17A, HSPD1, CYTIP, SLAMF7, CD14, C7, CORO2A, PPARGC1B, LTB4R, CHGA, CD300A, TLR6, CD209, and $P 2 R Y 14$.

The risk model was built with the following factors:

$$
\begin{gathered}
\text { Riskscore }=-665^{*} \text { CD } 4+\text { Tcells }+6.2^{*} L E P+10.7^{*} \text { NOX } 4 \\
+-5.92^{*} \text { RETNLB }+-34.9^{*} \text { LAIR } 1+5.67^{*} I L 17 A+-32.1 \\
{ }^{*} \text { HSPD } 1+23^{*} \text { CYTIP }+-16.3^{*} \text { SLAMF }+-15.3^{*} \text { CD } 14 \\
+-3.37^{*} C 7+-19.5^{*} \text { CORO } 2 A+14.5^{*} \text { PPARGC1B }+ \\
5.91^{*} \text { LTB } 4 R+4.7^{*} \text { CHGA }+17.5^{*} \text { CD } 300 A+-15.2^{*} \text { TLR6 } \\
+16.8^{*} \text { CD } 209+-14.5^{*} P 2 R Y 14
\end{gathered}
$$

According to this risk score, samples in the test set were divided into high- and low-risk groups, and $\mathrm{K}-\mathrm{M}$ survival analysis showed that the risk score has significant relevance with prognosis. The survival time of patients in the low-risk group was much longer than those in the high-risk group $(p \leq 0.01)$ in the TCGA test set (Figure 6A) and the independent validation dataset (Figure 6B). The AUC values of the survival time (113-/5-year survival rate) in TCGA test set predicted by this risk model were $0.733,0.728$, and 0.711 , respectively (Figure 6C). 

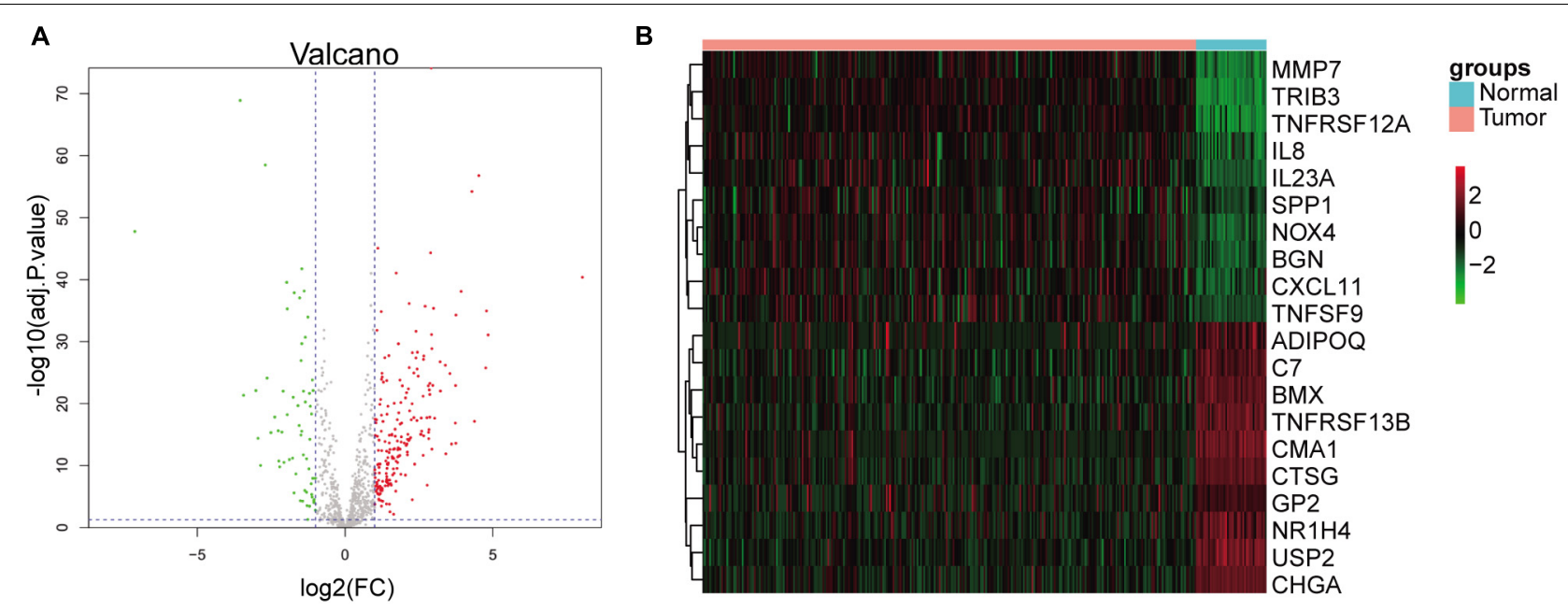

FIGURE 1 | Identification of differentially expressed immune genes. (A) Volcano plots of differentially expressed immune genes. Red dots represent upregulated genes, and green dots represent downregulated genes. (B) Heatmap of top 10 up- and downregulated immune genes.

\section{Nomogram Visualized Prognostic Model}

There were 127 samples with clinical number of lymph nodes and TNM stage in the test set. Based on the risk score of the risk model of multivariate Cox regression and prognostic clinical factors (lymph node examined count, pathologic $\mathrm{M}$, and pathologic $\mathrm{T}$ ), we visualized the nomogram of 127 test samples to show the risk model, as in Figure 7.

The results of the four prognostic factors in the nomogram and the $C$ index and $p$-value of combined model for the Coxph model are shown in Table 2. The results showed that the $C$ index of the combined model was the highest, and the risk score $(C$ index $>0.7$ ) had high fitting degree for the Coxph model. Among the clinical factors, only pathologic $M$ had the most significant statistical test result $(p=0.0108)$.

\section{DISCUSSION}

In the tumor stroma, there exists a complex biological process between immune cells and malignant cells, and the immune system plays a dual role of promoting and inhibiting tumor growth, which is of great significance for prognosis (Nagalla et al., 2013). In this study, we identified hundreds of immune genes and analyzed the tumor-infiltrating immune cells in colon cancer. Based on these immune genes and immune cells, we constructed and validated a colon cancer diagnostic prediction model and a prognostic model. B cell infiltration ratio, TRIB3, CHGA, CASP7, and multiple LGALS family genes such as LGALS4 were important features in diagnostic prediction model. Additionally, 19 factors such as CD4+ T cells, LEP, NOX4, IL17A, HSPD1, and CHGA had great influence on prognostic model.

In colorectal cancer, immune cells have significant infiltration, and their distribution, tissue localization, and cell types are highly relevant with progression and survival (Huh et al., 2012). Additionally, a study has reported that high infiltration of tumorinfiltrating immune cells in rectal cancer biopsies is related to improved tumor response to preoperative radiotherapy and chemotherapy, also with prolonged disease-free survival and OS (Anitei et al., 2014). In this study, B cell infiltration was found to be a diagnostic predictive feature of colon cancer, and $\mathrm{CD}^{+} \mathrm{T}$ cell infiltration was highly relevant with prognosis of colon cancer. A previous study has reported that infiltration of $\mathrm{CD}^{+}{ }^{+}$lymphocytes is frequent in colorectal cancer (Diederichsen et al., 2003). Our results further provided evidence that immune cell infiltration may represent a favorable prognostic factor of colon cancer.

In addition to immune cells, we also identified some diagnostic prediction-associated immune genes, such as TRIB3, CHGA, LGALS4, and CASP7. Tribbles pseudokinase 3 (TRIB3) is upregulated in some colorectal tumors and is responsible for poor outcome. TRIB3 can interact with $\beta$-catenin and transcription factor 4 in intestine cells to increase expression of genes that are relevant with cancer stem cells in colorectal cancer (Hua et al., 2019). Human chromogranin-A (CHGA) is a 439-residuelong protein found in the secretory granules of some normal and neoplastic neuroendocrine cells, the expression of which is related to the prognosis of colorectal cancer (Gunay et al., 2019). Long and Campbell (2017) reported that male patients with low expression of galactin 4 (LGALS4) had significantly shortened disease-free survival in colon cancer. Caspase-7 (CASP7) plays an important role in the autophagy and apoptosis of colon cancer (Athamneh et al., 2017). Additionally, CASP7 polymorphism is highly responsible for poor outcomes in patients with surgically resected colorectal cancer (Chae et al., 2011). Taken together, these genes may serve as diagnostic markers of colon cancer.

In this study, except for $\mathrm{CD}^{+}{ }^{+} \mathrm{T}$ cells, 18 important genes were identified in prognostic model, such as LEP, NOX4, IL17A, HSPD1, and CHGA. Leptin (LEP) is a cytokine produced by adipose tissue and plays a role in promoting tumorigenesis (Housa et al., 2006). A study has reported that LEP polymorphism is responsible for an increased risk of developing colorectal cancer (Partida-Pérez et al., 2010). NADPH oxidase 4 (NOX4) 


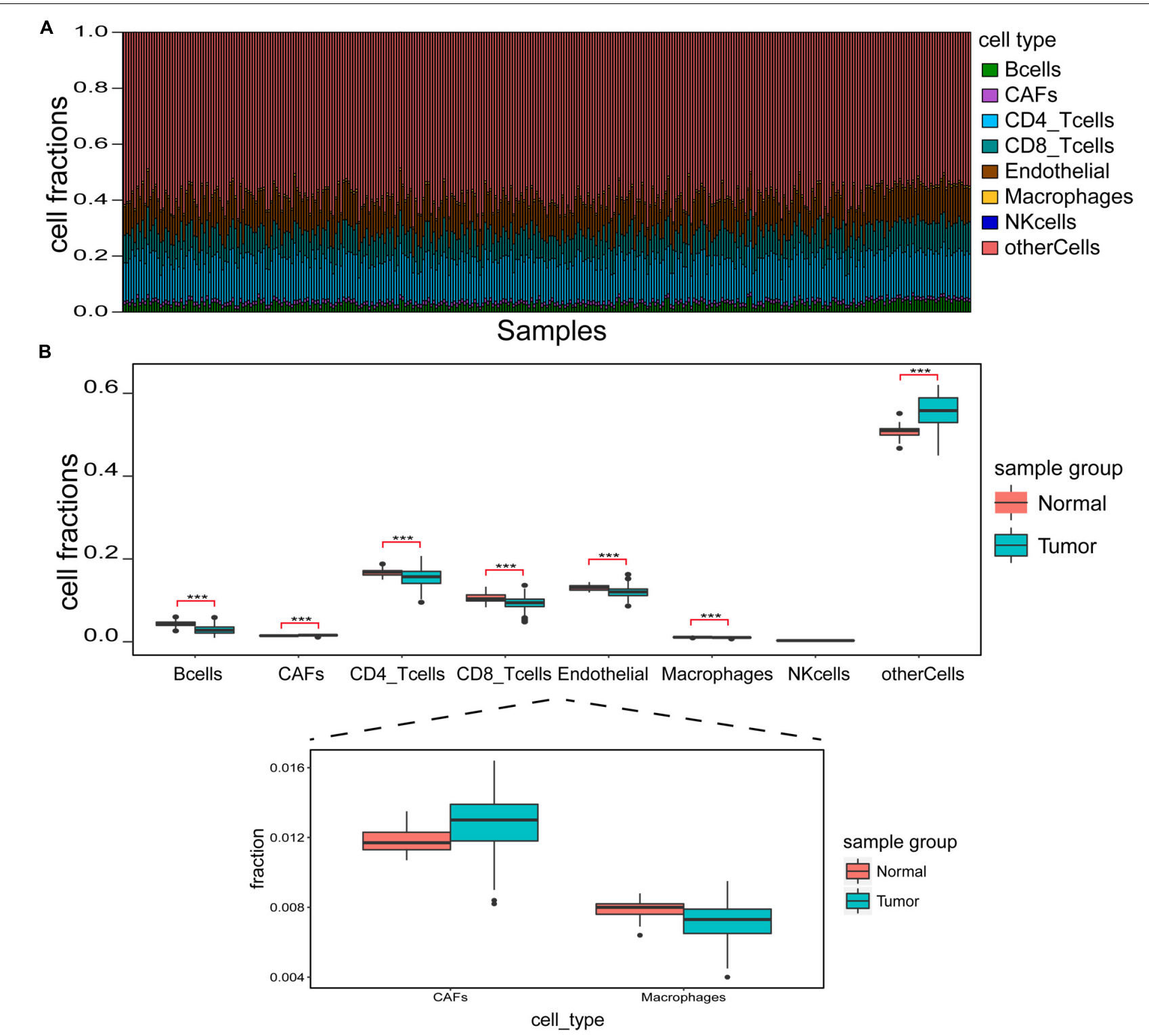

FIGURE 2 | Identification of immune cell infiltration features. (A) Immune cell infiltration ratio in each sample. (B) Differences in different immune cell components between tumor and normal samples. ${ }^{\star \star} p<0.001$.

is a major source of reactive oxygen species production, which has been reported to involve tumorigenesis (Juhasz et al., 2009). A recent study demonstrated that overexpression of NOX4 can promote tumor progression and predict poor prognosis in human colorectal cancer (Lin et al., 2017). IL17A is a cytokine that can be produced by some immune cells. It has been reported to play an important immunopathogenic role in inflammationrelated colonic diseases (Korn et al., 2009). Previous studies have reported that overexpression of IL17A means poor survival in colorectal cancer patients (Liu et al., 2011; Tosolini et al., 2011). Heat shock protein family D member 1 (HSPD1), as a signaling molecule in the immune system, is dysregulated in various cancers (Davalieva et al., 2015; Jin et al., 2016). Li et al. (2017) recently suggested that HSPD1 can serve as potential biomarker for the detection of colon cancer. Given the roles of these immune genes in colon cancer, we suppose that the constructed prognostic model may predict the prognosis of colon cancer.

\section{CONCLUSION}

In conclusion, our analysis constructed an immune-related prognostic model of colon cancer. B cell infiltration ratio, $\mathrm{CD} 4^{+}$ T cells, as well as genes of TRIB3, CHGA, CASP7, LGALS4, $L E P, N O X 4, I L 17 A$, and HSPD1 may be highly relevant with clinical outcome of colon cancer. Our results may help to 

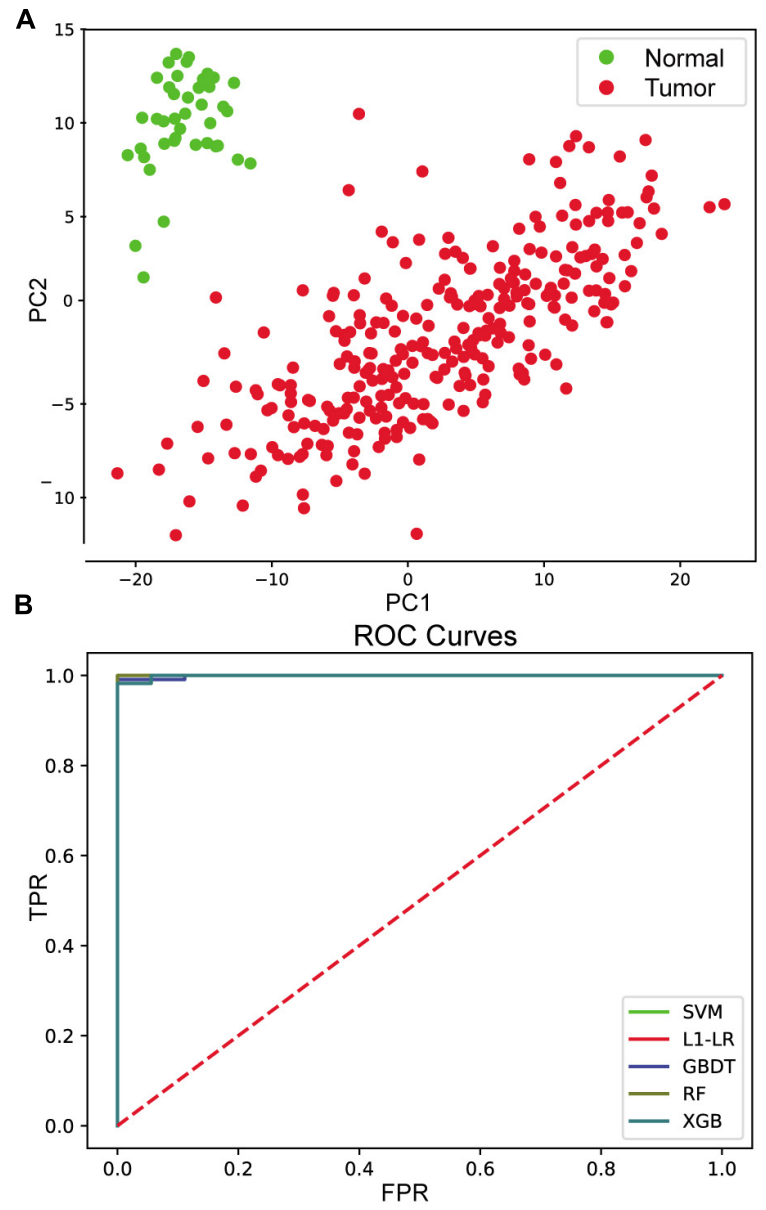

C

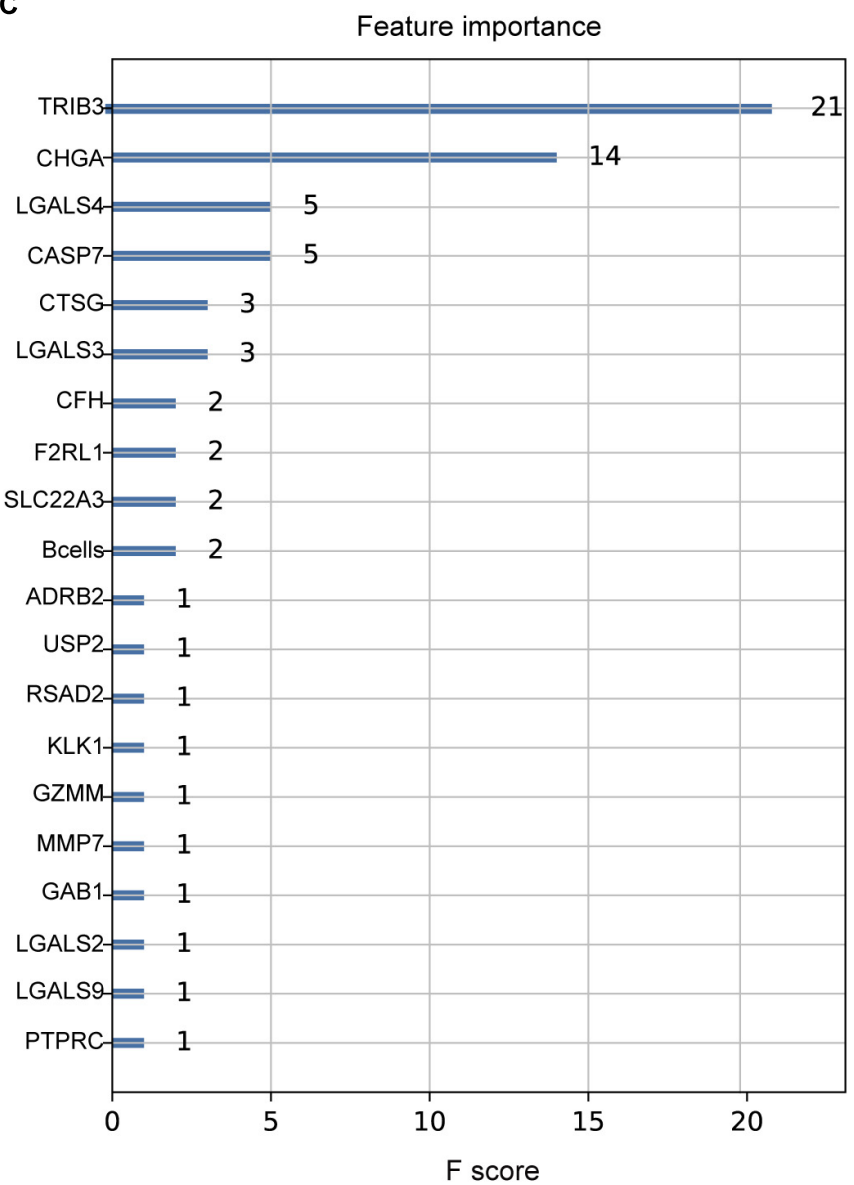

FIGURE 3 | Evaluation of colon cancer diagnostic model. (A) Principal component analysis (PCA) of two-dimensional visualization results. PC1 and PC2 represent the first two principal components with the largest variance in PCA results, respectively. (B) Receiver operating characteristic (ROC) curves of model. FPR represents false positive rate $[\mathrm{FP} /(\mathrm{FP}+\mathrm{TN})]$, and TPR represents true positive rate $[\mathrm{TP} /(\mathrm{TP}+\mathrm{FN})]$. The greater the area between ROC curve and $X$-axis (area under the curve value, between 0 and 1), the better the model effect. Different models are represented by different color curves. (C) The 20 most important features evaluated by XGB model. F score means the importance of the feature (the larger $F$ score means the greater contribution of the feature to the model).

TABLE 1 | The optimal parameters of different models and the evaluation results on the test set.

\begin{tabular}{|c|c|c|c|}
\hline Model & Parameter & Accuracy & Area under the curve \\
\hline SVM & Kernel = "rbf;" C = 1.0; degree = 3; cache_size = 200 & 0.992 & 1.0 \\
\hline L1-LogisticRegression & $\mathrm{C}=1.0 ;$ penalty = "11;" max_iter = 100; tol = 0.0001 & 1.0 & 1.0 \\
\hline GBDT & n_estimators $=50$; learning_rate $=0.1 ;$ max_depth $=3 ;$ subsample $=0.7 ;$ min_samples_split $=3$ & 0.985 & 0.996 \\
\hline RandomForest & n_estimators $=50 ;$ min_samples_leaf $=2 ;$ min_samples_split $=3$ & 1.0 & 1.0 \\
\hline XGBoost & max_depth $=3 ;$ min_child_weight $=1 ;$ gamma $=0.01$; learning_rate $=0.1 ;$ n_estimators $=50$ & 0.984 & 0.995 \\
\hline
\end{tabular}

uncover the clinical and biological significance of the immune microenvironment for colon cancer.

\section{MATERIALS AND METHODS}

\section{Gene Expression Data Collection}

The colon cancer gene expression profile data for the TCGA cohorts were downloaded from the University of California Santa Cruz (UCSC) Xena database (Crawshaw et al., 2007), which included gene expression data of 329 samples (288 tumor samples and 41 normal samples). The $\log 2(x+1)$-transformed RNA-Seq by Expectation-Maximization (RSEM) normalized read counts from UCSC Xena were downloaded for analysis. In addition, we also downloaded (download time: May 2019) the clinical phenotype data of the samples, including age, gender, TNM staging, and corresponding survival time and survival status. According to the gene annotation in the InnateDB database (Ge et al., 2002; Lang et al., 2016), 952 immune-related genes were annotated from the gene expression profile. 

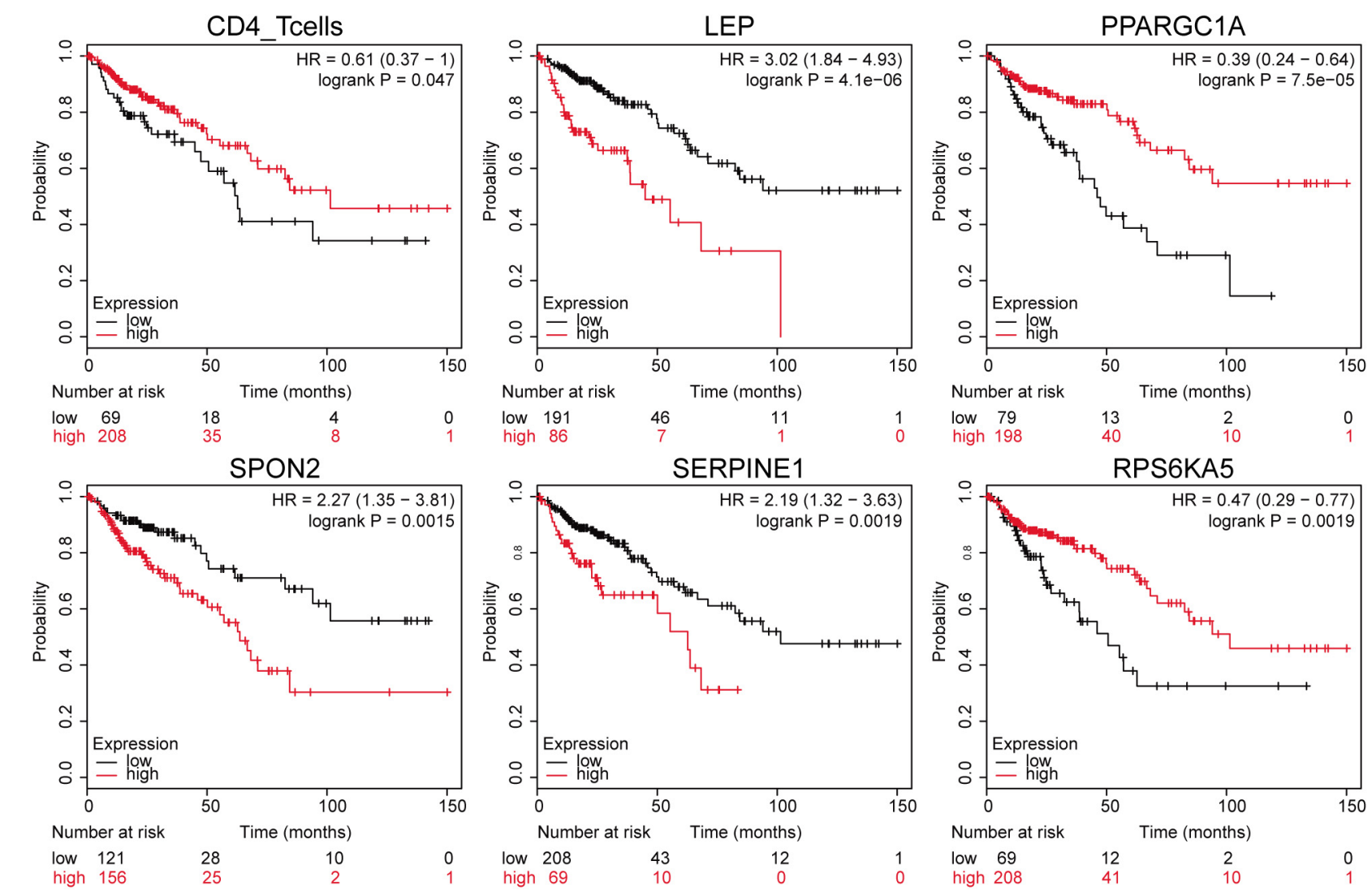

FIGURE 4 | Gene expression-related survival curve. The black and red curves represent the low- and the high-expression groups, respectively.

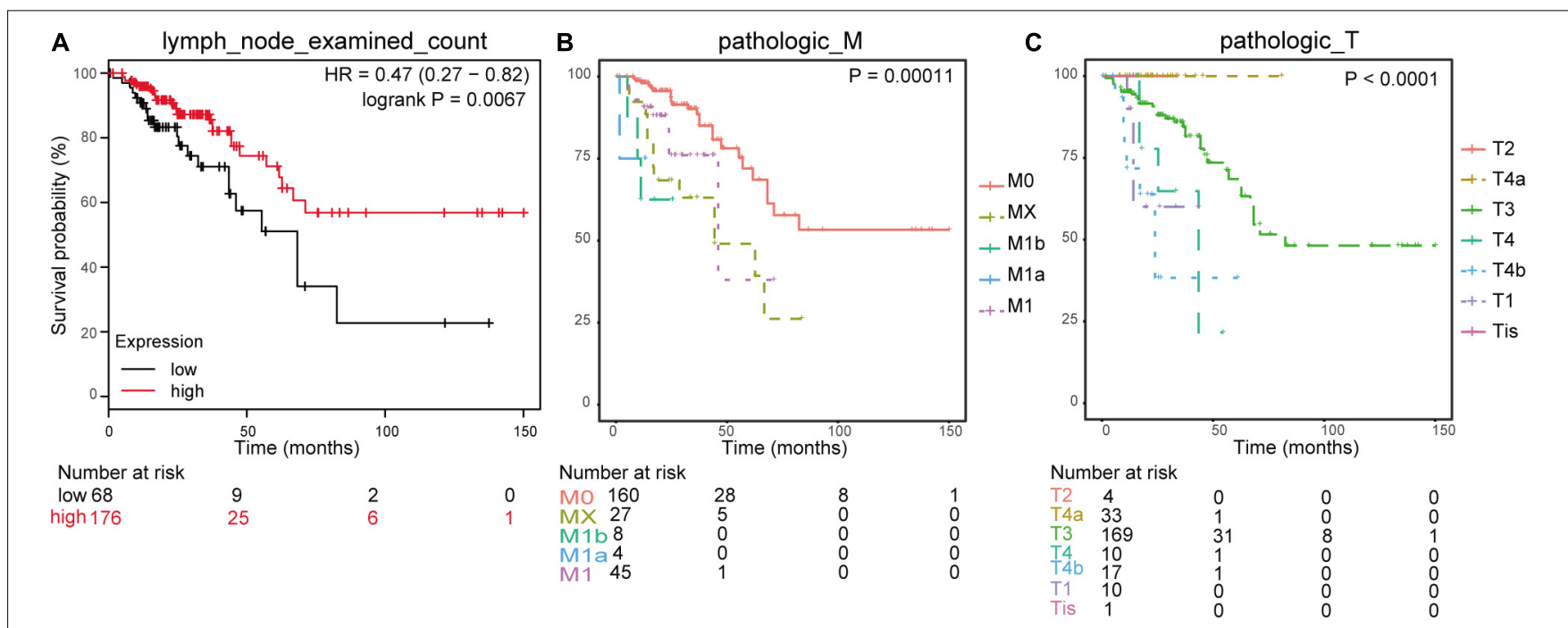

FIGURE 5 | Clinical phenotype-related survival curve. (A) Survival analysis for lymph node examined count. (B) Survival analysis for pathologic_M. (C) Survival analysis for pathologic_T.

For the validation dataset, gene expression dataset of GSE17538 (Smith et al., 2010) was downloaded from Gene Expression Omnibus (Barrett et al., 2013). This dataset included gene expression data of 244 colon cancer samples. After deleting the samples without survival information, 213 colon cancer samples and the corresponding survival information were obtained. The gene expression data have been preprocessed by robust multiarray average (RMA) normalization in affy 

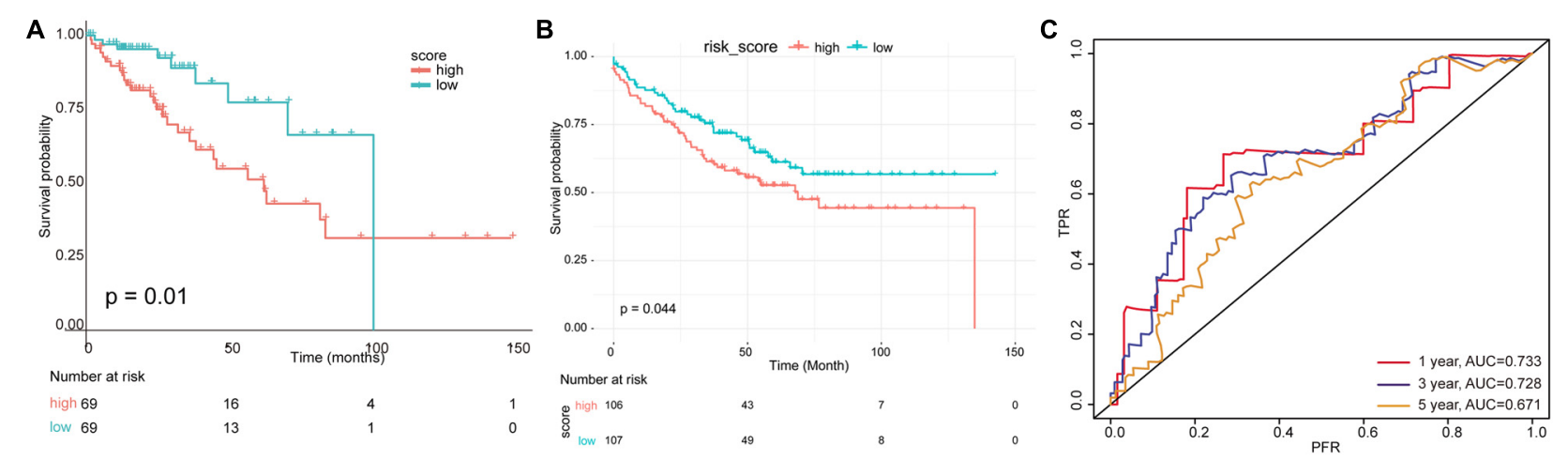

FIGURE 6 | Evaluation of multivariate prognostic model. (A) A risk model was constructed using 19 immune-related factors, and the test set was divided into highand low-risk groups according to scoring values. (B) The survival analysis for independent validation dataset. (C) The receiver operating characteristic (ROC) curve and area under the curve (AUC) value of survival time were predicted by multivariate risk model.

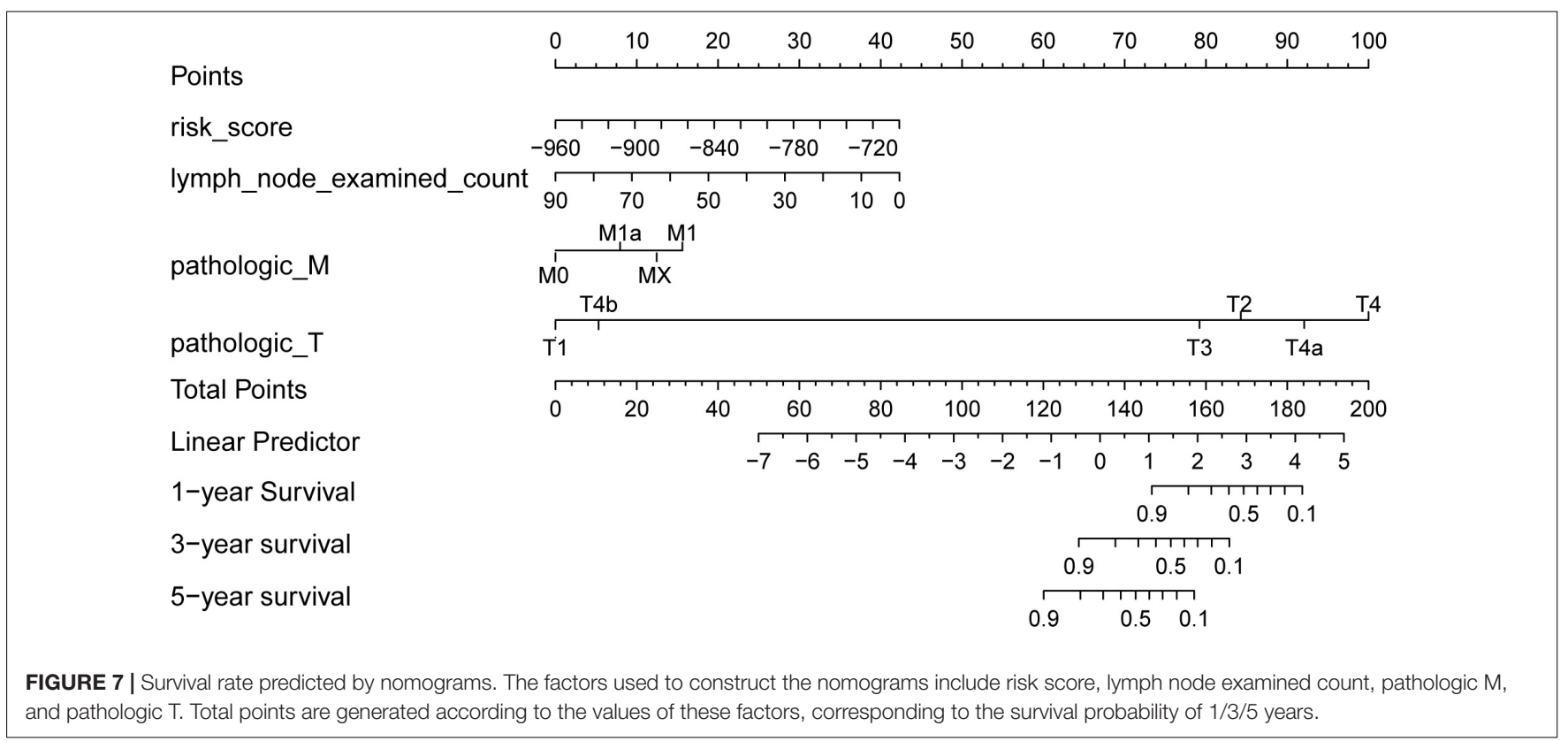

package. We downloaded the normalized gene expression data for further analysis.

\section{Differential Expression Analysis of Immune Genes}

For immune genes, the differential expression analysis for tumor vs. normal samples was performed using the limma package

TABLE 2 | Analysis of prognostic factors to the fitting degree of Coxph model.

\begin{tabular}{lcc}
\hline Factor & $\boldsymbol{C}$ index & $\boldsymbol{p}$-value \\
\hline Combined model & 0.828 & $3.22 E-21$ \\
Risk score & 0.725 & $4.16 E-11$ \\
Lymph node examined count & 0.587 & 0.0956 \\
Pathologic M & 0.636 & 0.0108 \\
Pathologic T & 0.596 & 0.0647
\end{tabular}

(Bradizza et al., 2006) (version 3.10.3). The genes with zero or missing values were removed. After statistical test, we obtained the corresponding $p$-values of all genes. Benjamini and Hochberg method was used for multiple test correction to obtain the adjusted $p$-value (adj. $p$-value). The genes with adj. $p$-value $<0.05$ and $\left|\log _{2} \mathrm{FC}\right|>1$ were selected as differentially expressed genes.

\section{EPIC Immune Cell Components Analysis}

Based on the gene expression profiles of 329 TCGA samples, we used immune cell infiltration analysis tools EPIC ${ }^{1}$ (Racle et al., 2017) to analyze the immune cells ratio in each sample, including B cells, CAFs, CD4 ${ }^{+} \mathrm{T}$ cells, CD8+ T cells, endothelial cells, macrophages, NK cells, and other types of cells. For the infiltration ratio of each type of immune cells, Welch two-sample

\footnotetext{
${ }^{1}$ https://gfellerlab.shinyapps.io/EPIC_1-1/
} 
non-paired $t$-test was used to test the differences between tumor and normal samples statistically.

\section{Diagnostic Model of Colon Cancer Based on Immune Characteristics}

The machine learning model was constructed in light of the immune cell components and differentially expressed gene information. Based on the integrated learning methods of GBDT, XGBoost, RandomForest, as well as the classification models of SVM and LR in Sklearn database ${ }^{2}$, we used the GridSearchCV package to build a model and adjust preferences. After adjustment and comparison of different models, we constructed an effective training classifier, which could predict colon cancer based on gene expression values. Here, we used the preprocessing.scale method to normalize the samples. Then, the samples were randomly divided into training and test sets at a ratio of 6:4 using train_test_split model from Sklearn database (random state $=123$ ). We used cross-validation to validate the training model and added the parameter of class_weight = "balanced" during the training to eliminate the influence of category imbalance. Finally, model evaluation was carried out on test set based on accuracy and AUC (mainly based on AUC value).

\section{Gene Expression/Clinical Phenotype and Prognosis Analysis}

The prognosis information of corresponding patients was collected on the basis of the downloaded clinical data, including OS and OS status. Combining the differentially expressed immune genes and patient's clinical phenotype, which are the candidate features with the sample prognostic information, we conducted the Kaplan-Meier (K-M) survival analysis by dividing the patients into high and low-expression groups based on gene expression or phenotypes. We used log-rank test to calculate the $p$-value and preliminarily screened genes with $p<0.05$ to obtain genes and clinical phenotypes associated with prognosis.

\section{Prognostic Model of Colon Cancer Based on Immune Characteristics}

The TCGA data set was randomly divided into training and test sets at a ratio of 5:5 (random state $=123$ ). In the training set, we used the prognostic immune cell components and immune gene expression, as well as the Coxph method in $\mathrm{R}$ package "survival" to comprehensively consider the effects of these characteristics and obtain the correlation significance $[\operatorname{Pr}(>|z|)]$ and coefficient (coef) of each feature. Here, we screened the genes with significance $p<0.05$. We used the screened significant genes and constructed the following multivariate risk model:

Risk score $=\beta$ gene $1 \times$ exprgene $1+\beta$ gene $2 \times$

$$
\text { exprgene } 2+\ldots+\beta \text { geneN } \times \text { exprgene }
$$

where $\beta$ gene1, $\beta$ gene $2, \ldots \beta$,geneN represents the coefficient of each gene in a multifactor analysis.

${ }^{2}$ https://scikit-learn.org/
For each sample in TCGA test set and the validation dataset, we calculated the risk score according to this formula and divided the samples into high- and low-risk groups on the basis of the median value of risk score. Then, K$M$ survival analysis was performed. In addition, we used this risk score as the standard to predict the 1-/3-/5year survival rate of patients in TCGA test set, and the receiver operating curve (ROC) and AUC value were used for model evaluation.

\section{Validation of the Prognostic Model in Independent Dataset}

The EPIC was used to analyze the immune cells ratio in each sample of GSE17538, including B cells, CAFs, CD4 ${ }^{+}$ $\mathrm{T}$ cells, $\mathrm{CD}^{+} \mathrm{T}$ cells, endothelial cells, macrophages, NK cells, and other types of cells. Risk score of each sample in validation dataset was calculated based on the following formula:

$$
\begin{gathered}
\text { Riskscore }=-665^{*} \text { CD } 4+\text { Tcells }+6.2^{*} \text { LEP }+10.7^{*} \text { NOX } 4 \\
+-5.92^{*} \text { RETNLB }+-34.9^{*} \text { LAIR } 1+5.67^{*} \text { IL } 17 A+-32.1 \\
{ }^{*} \text { HSPD } 1+23^{*} \text { CYTIP }+-16.3^{*} \text { SLAMF }+-15.3^{*} \text { CD } 14 \\
+-3.37^{*} C 7+-19.5^{*} \text { CORO2A }+14.5^{*} \text { PPARGC } 1 B+5.91 \\
{ }^{*} \text { LTB } 4 R+4.7^{*} \text { CHGA }+17.5^{*} \text { CD } 300 A+-15.2^{*} \text { TLR } 6 \\
+16.8^{*} \text { CD } 209+-14.5^{*} P 2 R Y 14
\end{gathered}
$$

All samples were divided into high- and low-risk groups according to the median value of the risk score. K-M survival analysis and log-rank test were performed for samples in highand low-risk groups.

\section{Nomogram Visualized Prognostic Model}

The survival rate could finally be obtained by mapping different factors to points and then adding them together. Therefore, on the basis of prognostic model risk score and prognostic clinical phenotypes, the nomogram prediction model was constructed and visualized. In order to further verify the predictive power of nomogram, we first calculated the independent prognostic factors and composite factors in the nomogram to fit the consistency index ( $C$ index) of Coxph model. On top of that, resampling method was used to carry out statistical test to calculate the $p$-value and compare the fitting degree of each independent prognostic and composite factors to the Coxph model.

\section{DATA AVAILABILITY STATEMENT}

The raw data supporting the conclusion of this article will be made available by the authors, without undue reservation, to any qualified researcher. 


\section{AUTHOR CONTRIBUTIONS}

All authors listed have made a substantial, direct and intellectual contribution to the work, and approved it for publication.

\section{FUNDING}

This work was supported by the National Natural Science Foundation of China under Grant Nos. 81874468 and 81403399. And also this study was supported by " 3 year plan of action to further accelerate the development

\section{REFERENCES}

Anitei, M.-G., Zeitoun, G., Mlecnik, B., Marliot, F., Haicheur, N., Todosi, A.-M., et al. (2014). Prognostic and predictive values of the immunoscore in patients with rectal cancer. Clin. Cancer Res. 20, 1891-1899. doi: 10.1158/1078-0432. CCR-13-2830

Athamneh, K., Hasasna, H. E., Samri, H. A., Attoub, S., Arafat, K., Benhalilou, N., et al. (2017). Rhus coriaria increases protein ubiquitination, proteasomal degradation and triggers non-canonical Beclin-1-independent autophagy and apoptotic cell death in colon cancer cells. Sci. Rep. 7:11633. doi: 10.1038/s41598017-11202-3

Barrett, T., Wilhite, S. E., Ledoux, P., Evangelista, C., Kim, I. F., Tomashevsky, M., et al. (2013). NCBI GEO: archive for functional genomics data sets-update. Nucleic Acids Res. 41, D991-D995. doi: 10.1093/nar/gks1193

Ben-Baruch, A. (2002). Host microenvironment in breast cancer development: inflammatory cells, cytokines and chemokines in breast cancer progression: reciprocal tumor-microenvironment interactions. Breast Cancer Res. 5:31. doi: $10.1186 /$ bcr554

Bense, R. D., Sotiriou, C., Piccart-Gebhart, M. J., Haanen, J. B., van Vugt, M. A., de Vries, E. G., et al. (2017). Relevance of tumorinfiltrating immune cell composition and functionality for disease outcome in breast cancer. JNCI J. Natl. Cancer Inst. 109:djw192. doi: 10.1093/jnci/dj w192

Bradizza, C. M., Stasiewicz, P. R., and Paas, N. D. (2006). Relapse to alcohol and drug use among individuals diagnosed with co-occurring mental health and substance use disorders: a review. Clin. Psychol. Rev. 26, 162-178. doi: 10.1016/j.cpr.2005.11.005

Bremnes, R. M., Al-Shibli, K., Donnem, T., Sirera, R., Al-Saad, S., Andersen, S., et al. (2011). The role of tumor-infiltrating immune cells and chronic inflammation at the tumor site on cancer development, progression, and prognosis: emphasis on non-small cell lung cancer. J. Thor. Oncol. 6, 824-833. doi: 10.1097/JTO. 0b013e3182037b76

Brockhoff, G., Seitz, S., Weber, F., Zeman, F., Klinkhammer-Schalke, M., Ortmann, O., et al. (2018). The presence of PD-1 positive tumor infiltrating lymphocytes in triple negative breast cancers is associated with a favorable outcome of disease. Oncotarget 9:6201. doi: 10.18632/oncotarget.23717

Chae, Y. S., Kim, J. G., Sohn, S. K., Lee, S. J., Kang, B. W., Moon, J. H., et al. (2011). RIPK1 and CASP7 polymorphism as prognostic markers for survival in patients with colorectal cancer after complete resection. J. Cancer Res. Clin. Oncol. 137, 705-713. doi: 10.1007/s00432-010-0929-1

Crawshaw, M. A., Glaser, A. W., and Pacey, A. A. (2007). The use of pornographic materials by adolescent male cancer patients when banking sperm in the UK: legal and ethical dilemmas. Hum. Fertil. (Camb.) 10, 159-163. doi: 10.1080/ 14647270701418922

Davalieva, K., Kostovska, I. M., Kiprijanovska, S., Markoska, K., Kubelka-Sabit, K., Filipovski, V., et al. (2015). Proteomics analysis of malignant and benign prostate tissue by 2D DIGE/MS reveals new insights into proteins involved in prostate cancer. Prostate 75, 1586-1600. doi: 10.1002/pros.23034

Diederichsen, A. C., Hjelmborg, J. V. B., Christensen, P. B., Zeuthen, J., and Fenger, C. (2003). Prognostic value of the $\mathrm{CD} 4^{+} / \mathrm{CD} 8+$ ratio of tumour infiltrating lymphocytes in colorectal cancer and HLA-DR expression on tumour cells. Cancer Immunol. Immunother. 52, 423-428. doi: 10.1007/s00262-003-0388-5 of traditional Chinese medicine in Shanghai" under Grant ZY(2018-2020)-CCCX-2004-09 and Peak Research Team Project in Shanghai University of Traditional Chinese Medicine.

\section{ACKNOWLEDGMENTS}

The results shown here are in part based upon data generated by the TCGA Research Network: https://www.cancer.gov/tcga. We thank Huaping Liu for critical reading of this manuscript and providing helpful suggestions in data processing.

Forman, D., Ferlay, J., Stewart, B., and Wild, C. (2014). The global and regional burden of cancer. World Cancer Rep. 2014, 16-53. doi: 10.1136/oemed-2019106012

Fridman, W. H., Pagès, F., Sautès-Fridman, C., and Galon, J. (2012). The immune contexture in human tumours: impact on clinical outcome. Nat. Rev. Cancer 12:298. doi: $10.1038 / \mathrm{nrc} 3245$

Galon, J., Costes, A., Sanchez-Cabo, F., Kirilovsky, A., Mlecnik, B., Lagorce-Pagès, C., et al. (2006). Type, density, and location of immune cells within human colorectal tumors predict clinical outcome. Science 313, 1960-1964. doi: 10. 1126/science.1129139

Ge, Y., Wang, X., Chen, Z., Landman, N., Lo, E. H., and Kang, J. X. (2002). Gene transfer of the Caenorhabditis elegans n-3 fatty acid desaturase inhibits neuronal apoptosis. J. Neurochem. 82, 1360-1366. doi: 10.1046/j.1471-4159. 2002.01077.x

Gunay, F. S. D., Kırm $\iota z \imath$, B. A., Ensari, A., Ýcli, F., and Akbulut, H. (2019). Tumor-associated macrophages and neuroendocrine differentiation decrease the efficacy of bevacizumab plus chemotherapy in patients with advanced colorectal cancer. Clin. Color. Cancer 18, e244-e250. doi: 10.1016/j.clcc.2018. 12.004

Housa, D., Housova, J., Vernerova, Z., and Haluzik, M. (2006). Adipocytokines and cancer. Physiol. Res. 55, 233-244.

Hua, F., Shang, S., Yang, Y. W., Zhang, H. Z., Xu, T. L., Yu, J. J., et al. (2019). TRIB3 Interacts With beta-Catenin and TCF4 to Increase Stem Cell Features of Colorectal Cancer Stem Cells and Tumorigenesis. Gastroenterology 70:e715. doi: 10.1053/j.gastro.2018.10.031

Huh, J. W., Lee, J. H., and Kim, H. R. (2012). Prognostic significance of tumorinfiltrating lymphocytes for patients with colorectal cancer. Arch. Surg. 147, 366-372. doi: 10.1001/archsurg.2012.35

Jin, H., Cheng, X., Pei, Y., Fu, J., Lyu, Z., Peng, H., et al. (2016). Identification and verification of transgelin-2 as a potential biomarker of tumor-derived lungcancer endothelial cells by comparative proteomics. J. Proteomics 136, 77-88. doi: 10.1016/j.jprot.2015.12.012

Juhasz, A., Ge, Y., Markel, S., Chiu, A., Matsumoto, L., Van Balgooy, J., et al. (2009). Expression of NADPH oxidase homologues and accessory genes in human cancer cell lines, tumours and adjacent normal tissues. Free Radical Res. 43, 523-532. doi: 10.1080/10715760902918683

Kim, Y., Hoehn, H. J., Chen, Y., Barnard, M. E., Bloomer, A., Yoder, S., et al. (2018). "Prognostic gene expression signatures of immune responses in the colon cancer microenvironment," in Proceedings of the AACR Annual Meeting 2018, Chicago, IL.

Korn, T., Bettelli, E., Oukka, M., and Kuchroo, V. K. (2009). IL-17 and Th17 Cells. Ann. Rev. Immunol. 27, 485-517. doi: 10.1146/annurev.immunol.021908. 132710

Lang, J., Gonzalez-Mula, A., Taconnat, L., Clement, G., and Faure, D. (2016). The plant GABA signaling downregulates horizontal transfer of the Agrobacterium tumefaciens virulence plasmid. New Phytol. 210, 974-983. doi: 10.1111/nph. 13813

Li, G., Li, M., Liang, X., Xiao, Z., Zhang, P., Shao, M., et al. (2017). Identifying DCN and HSPD1 as potential biomarkers in colon cancer using 2D-LC-MS/MS combined with iTRAQ technology. J. Cancer 8:479. doi: 10.7150/jca.17192

Lin, X.-L., Yang, L., Fu, S.-W., Lin, W.-F., Gao, Y.-J., Chen, H.-Y., et al. (2017). Overexpression of NOX4 predicts poor prognosis and promotes tumor 
progression in human colorectal cancer. Oncotarget 8:33586. doi: 10.18632/ oncotarget.16829

Liu, J., Duan, Y., Cheng, X., Chen, X., Xie, W., Long, H., et al. (2011). IL-17 is associated with poor prognosis and promotes angiogenesis via stimulating VEGF production of cancer cells in colorectal carcinoma. Biochem. Biophys. Res. Commun. 407, 348-354. doi: 10.1016/j.bbrc.2011.03.021

Long, M. D., and Campbell, M. J. (2017). Integrative genomic approaches to dissect clinically-significant relationships between the VDR cistrome and gene expression in primary colon cancer. J. Steroid Biochem. Mol. Biol. 173, 130-138. doi: 10.1016/j.jsbmb.2016.12.013

Nagalla, S., Chou, J. W., Willingham, M. C., Ruiz, J., Vaughn, J. P., Dubey, P., et al. (2013). Interactions between immunity, proliferation and molecular subtype in breast cancer prognosis. Genome Biol. 14:R34. doi: 10.1186/gb-2013-14-4-r34

Partida-Pérez, M., de la Luz Ayala-Madrigal, M., Peregrina-Sandoval, J., Macías-Gómez, N., Moreno-Ortiz, J., Leal-Ugarte, E., et al. (2010). Association of LEP and ADIPOQ common variants with colorectal cancer in Mexican patients. Cancer Biomarkers 7, 117-121. doi: 10.3233/CBM-20100154

Racle, J., de Jonge, K., Baumgaertner, P., Speiser, D. E., and Gfeller, D. (2017). Simultaneous enumeration of cancer and immune cell types from bulk tumor gene expression data. eLife 6:e26476. doi: 10.7554/eLife.26476

Shibutani, M., Maeda, K., Nagahara, H., Fukuoka, T., Iseki, Y., Matsutani, S., et al. (2018). Tumor-infiltrating lymphocytes predict the chemotherapeutic outcomes in patients with stage IV colorectal cancer. In Vivo 32, 151-158. doi: 10.21873 /invivo.11218
Siegel, R. L., Miller, K. D., Fedewa, S. A., Ahnen, D. J., Meester, R. G., Barzi, A., et al. (2017). Colorectal cancer statistics, 2017. CA Cancer J. Clin. 67, 177-193.

Smith, J. J., Deane, N. G., Wu, F., Merchant, N. B., Zhang, B., Jiang, A., et al. (2010). Experimentally derived metastasis gene expression profile predicts recurrence and death in patients with colon cancer. Gastroenterology 138, 958-968. doi: 10.1053/j.gastro.2009.11.005

Tosolini, M., Kirilovsky, A., Mlecnik, B., Fredriksen, T., Mauger, S., Bindea, G., et al. (2011). Clinical impact of different classes of infiltrating $\mathrm{T}$ cytotoxic and helper cells (Th1, th2, treg, th17) in patients with colorectal cancer. Cancer Res. 71, 1263-1271. doi: 10.1158/0008-5472.CAN-10-2907

Udall, M., Rizzo, M., Kenny, J., Doherty, J., Dahm, S., Robbins, P., et al. (2018). PD-L1 diagnostic tests: a systematic literature review of scoring algorithms and test-validation metrics. Diagnostic Pathol. 13:12. doi: 10.1186/s13000-0180689-9

Conflict of Interest: The authors declare that the research was conducted in the absence of any commercial or financial relationships that could be construed as a potential conflict of interest.

Copyright (c) 2020 Yang, Jin, Liu, Gan, Cui, Han and Wang. This is an open-access article distributed under the terms of the Creative Commons Attribution License (CC BY). The use, distribution or reproduction in other forums is permitted, provided the original author(s) and the copyright owner(s) are credited and that the original publication in this journal is cited, in accordance with accepted academic practice. No use, distribution or reproduction is permitted which does not comply with these terms. 